\title{
Merging Standard CVD techniques for GaAs and Si Epitaxial Growth
}

\author{
A. Sammak, W. de Boer, A. van den Bogaard and L.K. Nanver \\ Delft Institute of Microsystems and Nanoelectronics (DIMES), Delft University of \\ Technology, Delft, The Netherlands
}

\begin{abstract}
A commercial Chemical Vapor Deposition (CVD) system, the ASMI Epsilon 2000 designed for Si and SiGe epitaxy, has, for the first time, been equipped for the growth of GaAs compounds in a manner that does not exclude the use of the system also for Sibased depositions. With the new system, intrinsic, Si-doped and Ge-doped GaAs epitaxial layers with excellent quality have been grown on GaAs substrate wafers by the decomposition of trimethylgallium (TMGa) and $\mathrm{AsH}_{3}$ in the reactor at reduced pressure and at temperatures in the $600-700^{\circ} \mathrm{C}$ range. A low $\mathrm{AsH}_{3}$ concentration, $0.7 \%$ in $\mathrm{H}_{2}$, is used as one of the precursors, which has the added advantage that the severe safety precautions always associated with MOCVD systems need not be implemented.
\end{abstract}

\section{Introduction}

This paper presents details and conditions of GaAs epitaxial growth on GaAs substrates in an initially $\mathrm{Si} / \mathrm{SiGe}$ based Chemical Vapor Deposition (CVD) system. GaAs is potentially of great importance for many high-speed electronics and optoelectronics devices (1). It is recognized as a material with a large electron mobility that is suitable for high-speed electronics and with a direct band-gap that is perfectly applicable for fabrication of light emitting diodes (LEDs), laser diodes (LDs), photodetectors and waveguides. Now, GaAs is the basis of several billion dollar worldwide industry for high-frequency, high-speed electronics and optoelectronics and GaAs epitaxial techniques are playing important roles in this industry. The use of such techniques allows more flexibility in the growth and placement of semiconducting layers and dopants than is achieved by other approaches such as bulk crystal growth, ion implantation and diffusion (2). In the last few years, different CVD methods for epitaxial growth have been developed to such high level that excellent thin GaAs layers can be grown with good quality and growth rate control (3-8). However, all these methods use reactors that are specially designed for GaAs and other III-V families, usually not compatible with Si processing. So, the ability of merging standard CVD techniques for III-V semiconductors and Si epitaxy in one single reactor is completely new and could be of great interest for integration of III-V materials and Si devices. For this purpose, a standard commercial $\mathrm{Si} / \mathrm{SiGe}$ CVD system has been modified to allow the deposition of III-V semiconductors such as GaAs and GaN in addition to the standard Si and SiGe depositions. With the new system, excellent quality GaAs epitaxial layers have been grown on GaAs substrate wafers by the decomposition of TMGa and $\mathrm{AsH}_{3}$ in the reactor at reduced pressure and at temperatures in the $600-700^{\circ} \mathrm{C}$ range. Also Si and Ge doping profiles of the GaAs during epitaxy are attainable by using the methylsilane $\left(\mathrm{CH}_{3} \mathrm{SiH}_{3}\right)$ and germane $\left(\mathrm{GeH}_{4}\right)$ gas lines that are already standard accessories of the Si/SiGe epitaxy equipment. 


\section{Description of the System}

This process is carried out in an ASM Epsilon 2000, 100 mm CVD system, mainly used for the epitaxial growth of $\mathrm{Si}$ and $\mathrm{SiGe}$ alloys. The doping gases are $\mathrm{B}_{2} \mathrm{H}_{6}, \mathrm{PH}_{3}, \mathrm{AsH}_{3}$ and, for $\mathrm{SiGe}, \mathrm{SiH}_{3} \mathrm{CH}_{3}$. It is equipped with integrated heating lamps to grow layers at temperatures up to $1200^{\circ} \mathrm{C}$ at atmospheric and reduced pressures. The schematic diagram of the CVD reactor is shown in Fig.1. For chemical vapor deposition, a $100 \mathrm{~mm} \mathrm{Si}$ or GaAs wafer is located on top of a graphite susceptor which is rotating during the deposition process while the gas precursors flow parallel to the surface of the wafer. The system was extended with a trimethylgallium (TMGa) bubbler and an extra gas line to allow the deposition of III-V semiconductors such as GaAs and GaN in addition to the standard Si and SiGe depositions. Hydrogen carrier gas is bubbled through the TMGa and the ratio of process and carrier gas is sensed and controlled by a state-of-the-art Lorex Piezocon binary gas concentration sensor.

For the GaAs depositions a low arsine concentration is applied: $0.7 \%$ as compared to the at least ten times higher values normally used in MOCVD equipment. This has the advantage that the severe safety precautions always associated with MOCVD systems need not be implemented. The obvious effect of using a very low $\mathrm{AsH}_{3}$ concentration is the correspondingly low growth rate. Nevertheless, GaAs growth rates of 0.5 to $5 \mathrm{~nm} / \mathrm{min}$ have been achieved, with the growth rate increasing as the TMGa and $\mathrm{AsH}_{3}$ concentrations are increased. Such values are acceptable for many of today's device applications for which layers in the $100 \mathrm{~nm}$ range are required.

GaAs epitaxial growth on GaAs substrates is performed at a pressure of 80Torr and at temperatures ranging between $600^{\circ} \mathrm{C}$ and $700^{\circ} \mathrm{C}$. First, a baking step at $650^{\circ} \mathrm{C}$ is carried out in $\mathrm{H}_{2}$ ambient for 3 minutes in order to remove the native oxide. Then TMGa and $\mathrm{AsH}_{3}$ are introduced into the chamber with $\mathrm{AsH}_{3} / \mathrm{TMGa}$ ratios from 20 to 40 in the gas phase.

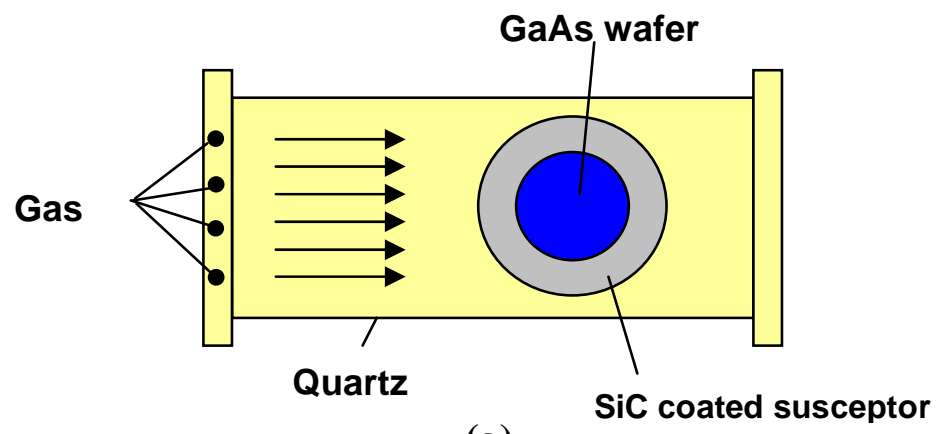

(a)

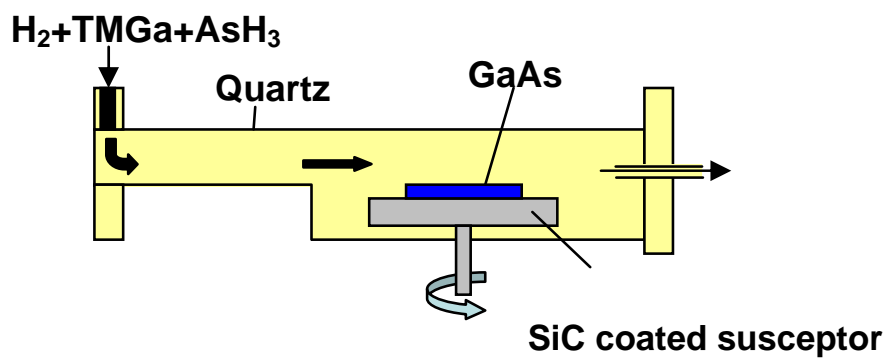

(b)

Figure 1. A schematic diagram of the (a) top view and (b) side view of the CVD reactor 


\section{Results and Discussions}

In order to utilize the CVD reactor for both GaAs and Si processings, after several GaAs growth cycles, the system can be cleaned by performing a few high temperature $\mathrm{HCl}$ cleaning steps. Si epitaxy with dopant concentrations as low as $10^{15} \mathrm{~cm}^{-3}$, is then ypossible to be grown. An example of a situation where the ability to grow Si and GaAs layers in the same system can be of advantage, is the growth of GaAs on Si substrates by using an intermediate SiGe buffer layer. Since the wafers can remain in the same reactor during the $\mathrm{SiGe/GaAs} \mathrm{growth} \mathrm{sequence,} \mathrm{there} \mathrm{is} \mathrm{no} \mathrm{need} \mathrm{for} \mathrm{cleaning} \mathrm{steps} \mathrm{in} \mathrm{between} \mathrm{the} \mathrm{two}$ layers to remove the native oxide.

The $\mathrm{AsH}_{3} / \mathrm{TMGa}$ ratio was found not to be very critical. Fig. 2 shows the plots from typical SIMS analysis carried out on GaAs epitaxial layers on GaAs substrates. The layers have been deposited with different $\mathrm{AsH}_{3} / \mathrm{TMGa}$ ratios. Ratios of 20, 30 and 40 have been investigated which proves that values from 20 - 40 in the gas phase all gives stoichiometric GaAs growth. However, different ratios still result in different growth rates.

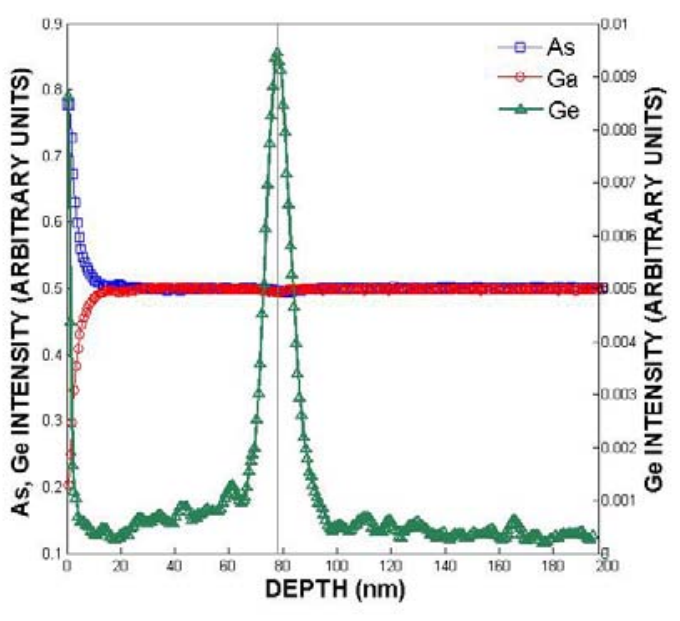

(a)

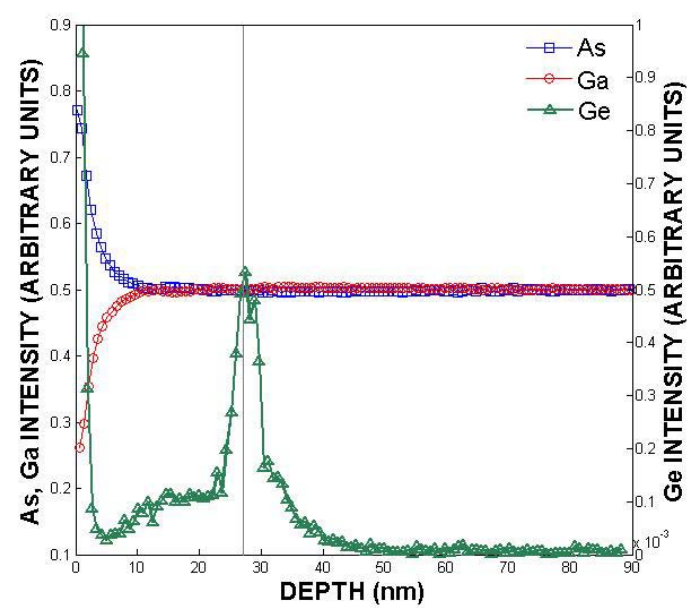

(b)

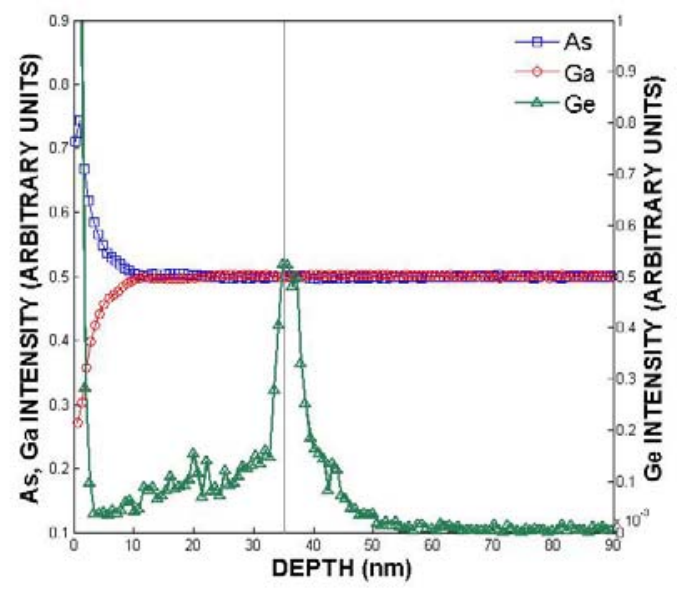

(c)

Figure 2. SIMS plots for GaAs epitaxial growth with $\mathrm{AsH}_{3} / \mathrm{TMGa}$ ratios of (a) 20, (b) 30 and (c) 40 in the gas phase with perfect stoichiometry. 
In all samples, a Ge marker has been used before commencing the GaAs growth so that the interface between the substrate and the epitaxial layer is accurately monitored by the SIMS.

An experiment in which the $\mathrm{AsH}_{3}$ and TMGa concentrations were varied while the $\mathrm{AsH}_{3} / \mathrm{TMGa}$ ratio was kept constant is shown in Fig. 3. Three layers were grown consecutively. A Ge marker indicates where a decrease in concentration is implemented as going towards the surface. The first layer with the highest gas flows has a growth rate of $5 \mathrm{~nm} / \mathrm{min}$; the second and third layers have rates of $3 \mathrm{~nm} / \mathrm{min}$ and $0.5 \mathrm{~nm} / \mathrm{min}$ respectively. The $\mathrm{AsH}_{3} / \mathrm{TMGa}$ ratio was set to be 30 and the stoichiometry of Ga and As, as shown in Fig. 3, is still perfect for all three cases with different gas flows and growth rates.

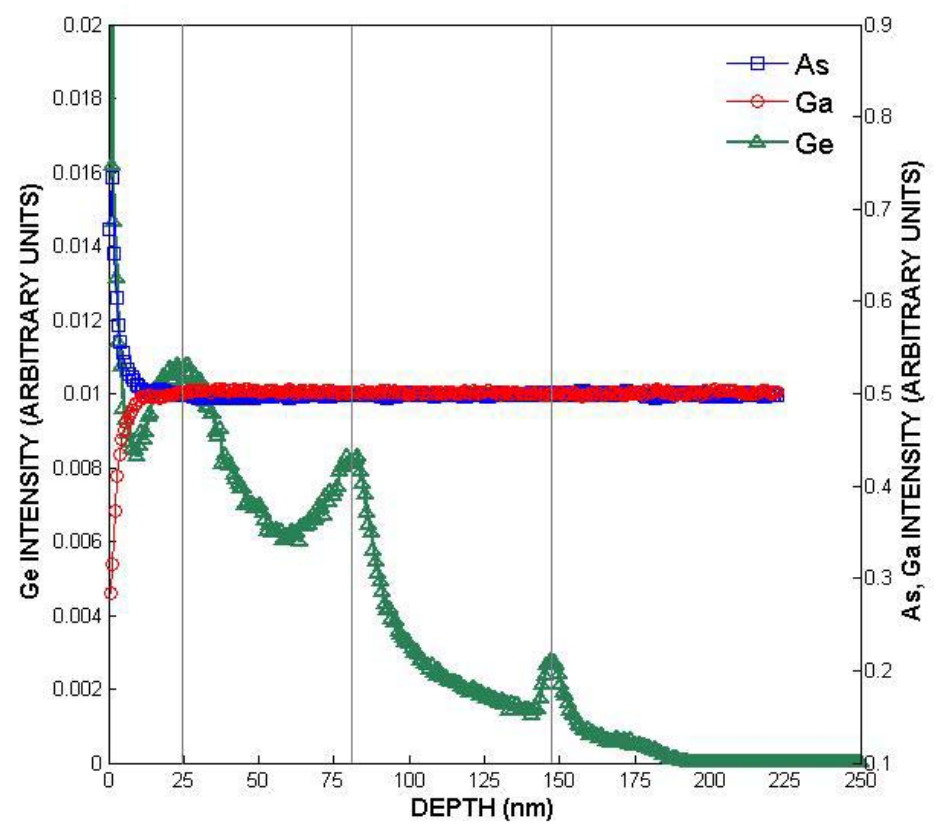

Figure 3. SIMS profile of three intrinsic GaAs epitaxial layers with growth rates of 5, 3 and $0.5 \mathrm{~nm} / \mathrm{min}$, respectively. The Ge peaks have been grown for the determination of interfaces between layers. The As and Ga concentrations in the substrate and in the epi layers are the same, indicating good stoichiometry in the deposited layers.

\section{$\underline{\text { Si and Ge-doped GaAs epitaxy }}$}

Si and Ge are well-known n-type impurities for GaAs. Their incorporation in GaAs when epitaxial growth is mainly based on replacing $\mathrm{Ga}$ in the lattice, which leads to ntype doping in GaAs $(9,10)$. Both Si and Ge-doped GaAs epitaxial layers have been achieved, using $0.1 \%$ methylsilane $\left(\mathrm{CH}_{3} \mathrm{SiH}_{3}\right)$ and $2 \%$ germane $\left(\mathrm{GeH}_{4}\right)$ gases respectively, in addition to the main GaAs precursors. The lines for these two gases are part of the standard equipment of the reactor.

Fig. 4 shows a SIMS profile of a $100 \mathrm{~nm}$ Si-doped GaAs epitaxial layer with a dopant concentration of $2 \times 10^{19} \mathrm{~cm}^{-3}$. It is interesting to point out that the $\mathrm{C}$ concentration in the epitaxial layer is lower than that of the substrate, in spite of the fact that methylsilane decomposition results in an introduction of the same amount of Si and C. Consequently 
methylsilane can be considered to be a good alternative for silane $\left(\mathrm{SiH}_{4}\right)$, the standard dopant precursor for Si-doped GaAs epitaxy.

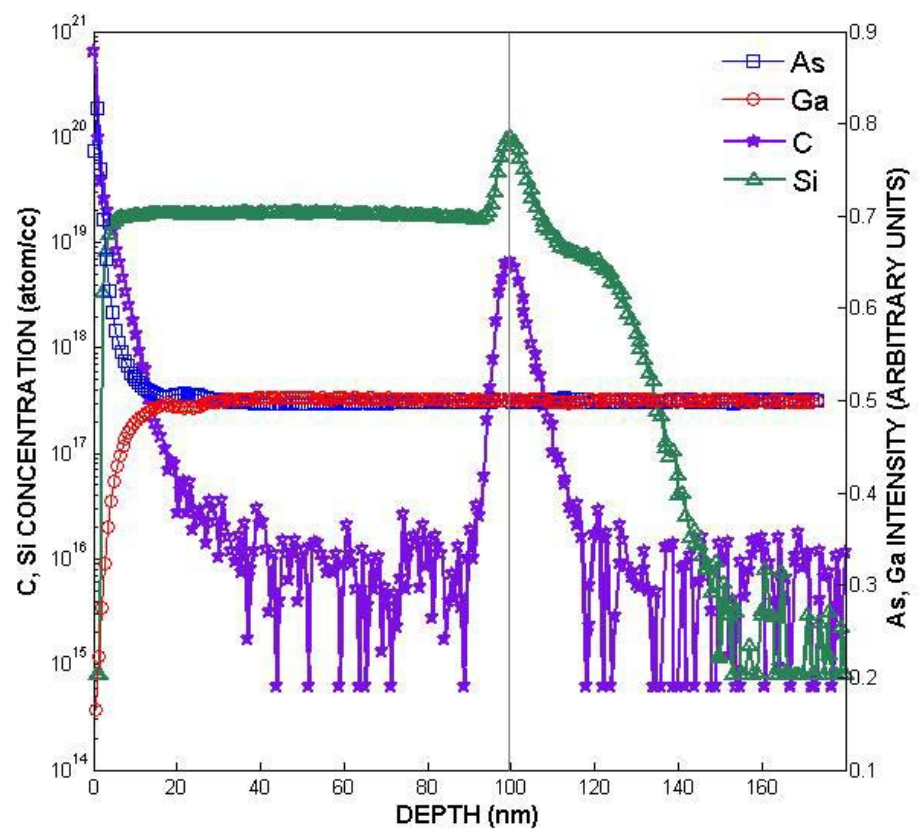

Figure 4. SIMS profile of a $2 \times 10^{19} \mathrm{~cm}^{-3} \mathrm{Si}$ dope in a stoichiometric GaAs epitaxial layer.

The Si spike at the interface in this, and other SIMS plots is most probably caused by the coating of the susceptor: before loading the GaAs wafer we apply a thin Si coating on the susceptor. It is possible that this coating causes a Si spike at the interface. The C spike, visible in some of the SIMS plots, is most probably due to insufficient cleaning of the surface before the deposition starts.

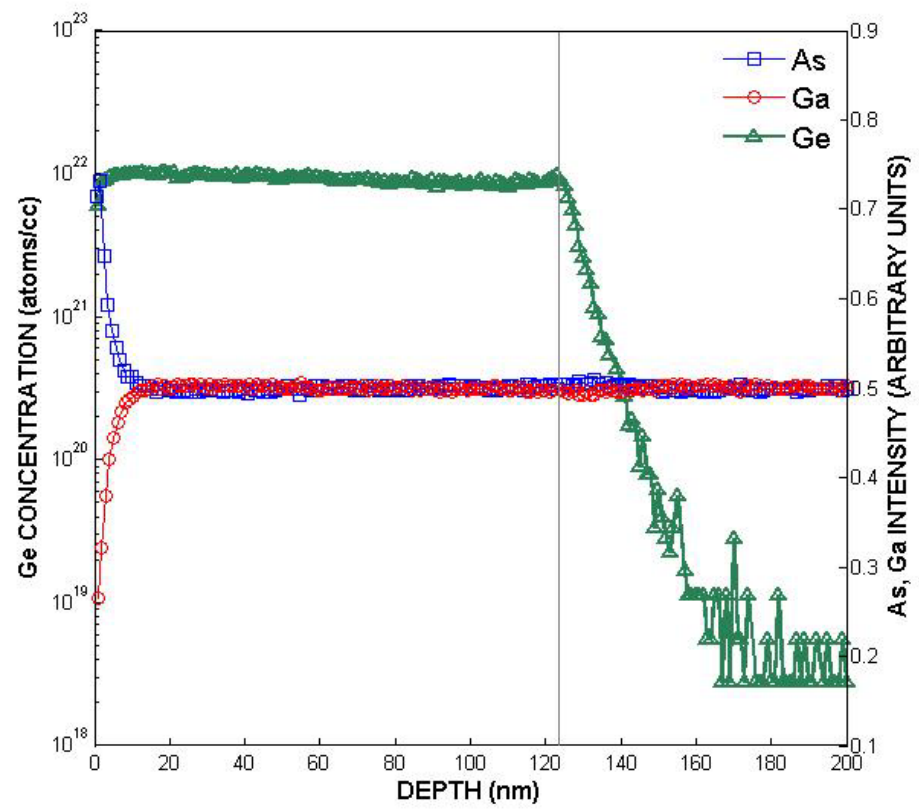

Figure 5. SIMS profile of a Ge doped GaAs epitaxial layer. At this high concentration Ge tends to replace Ga and As equally, at least within the accuracy of the measurement. 
Very high Ge-doped GaAs epitaxial layers with a uniform dopant concentration of $10^{22} \mathrm{~cm}^{-3}$ have been obtained, as shown in Fig. 5, while the GaAs stoichiometry is still ideal. At this high concentration, well over $20 \%$, the Ga and As concentrations still seem to be equal, within the SIMS accuracy. Hence, Ga and As are approximately equally replaced by $\mathrm{Ge}$ in this high concentration regime.

Using the reactor's dilution system, the concentration of the impurity precursors can be reduced, giving rise to dopant concentration control over several orders of magnitude. This is illustrated in Fig. 6, which shows a Si-doped and a Ge-doped epitaxial layer with an attempt to grow a staircase doping profile. Fig. 6a shows three Si doping levels with averages of approximately $5 \times 10^{17}, 10^{19}$ and $8 \times 10^{19} \mathrm{~cm}^{-3}$, respectively and Fig. $6 \mathrm{~b}$ is the profile for three Ge doping levels with averages of approximately $5 \times 10^{20}, 5 \times 10^{19}$ and $1 \times 10^{19} \mathrm{~cm}^{-3}$, respectively. However, the doping profile at the layer interfaces is not abrupt. This is because of the rather high diffusion coefficients of Si and Ge in GaAs in the 600$700^{\circ} \mathrm{C}$ temperature range. As is clearly visible, considerable diffusion has taken place. Not only are the changes in concentration far from abrupt, also both, Si and Ge, have diffused past the original interface into the substrate. It is also clear that the deeper layers have suffered more from diffusion as they have been exposed to a higher temperature budget.

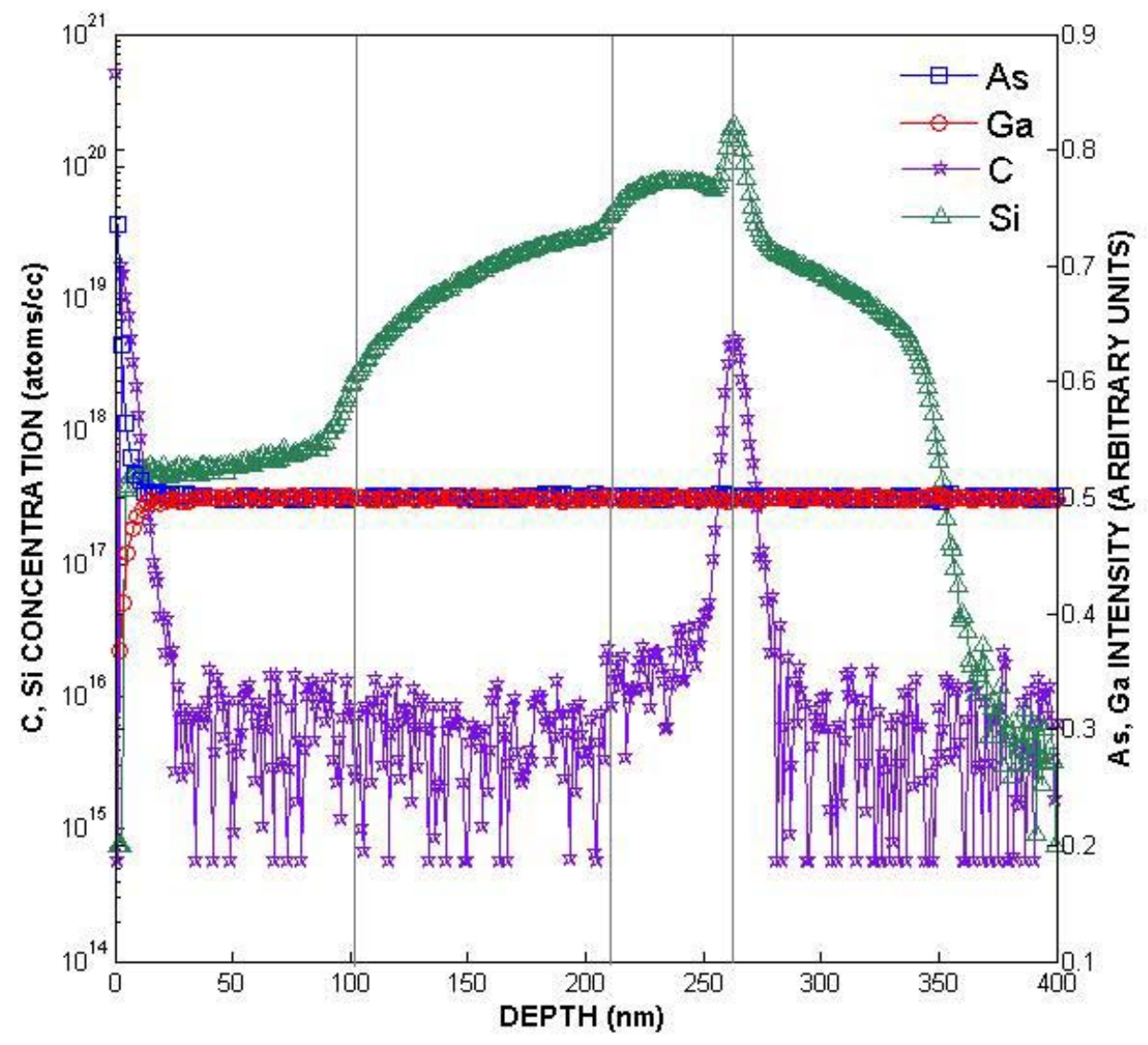

(a) 


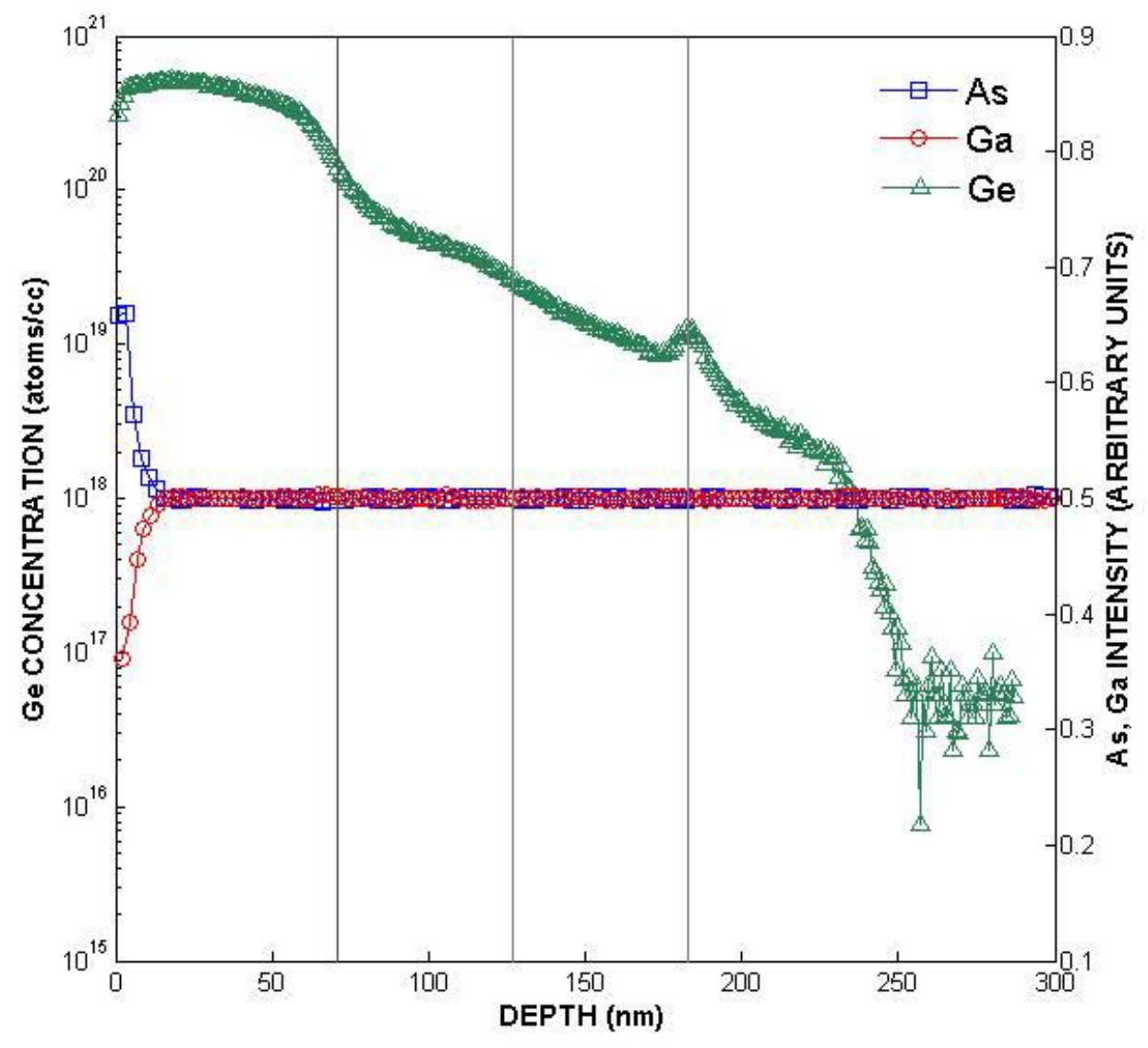

(b)

Figure 6. SIMS plots for (a) Si-doped and (b) Ge-doped staircase GaAs epitaxial layers

\section{Conclusions}

The epitaxial growth of GaAs in a commercial Si/SiGe CVD reactor has been demonstrated in a manner that is compatible with the standard Si/SiGe epitaxy. A good quality, stoichiometric GaAs is obtained for a wide range of deposition parameters and at useful growth rates. The background doping level of the reactor can quickly be returned to the range that is compatible with Silicon processing with standard cleaning methods. Moreover, doping with $\mathrm{Si}$ or Ge is readily obtained from standard gas sources.

\section{Acknowledgments}

The authors would like to gratefully acknowledge the DIMES-ICP group for processing support and the SmartMix Memphis project for financial support. 


\section{References}

1. S. Adachi, GaAs and Related Materials: Bulk Semiconducting and Superlattice Properties, p.1-5, World Scientific Publitiong Co., Singapore (1994).

2. A. G. Baca and C. I. H. Ashby, Fabrication of GaAs Devices, p. 38-40, The Institution of Electrical Engineers, London, (2005).

3. C. Pelosi, G. Attolini, M. Bosi, D. Moscatelli, A. Veneroni, M. Masi, J. Chrystal Growth, 287, p 652-655 (2006).

4. R. Ares, S. P. Watkins, P. Yeo, G. A. Horley, P. O’Brien and A. C. Jones, J. Applied Physics, 83, 6 (1998).

5. H. Beneking, A. Escobosa and H. Krautle, J. Electronic Materials, 10, 3 (1981).

6. N. Kobayashi, T. Fukui and Y. Horikoshi, Japanese J. Applied Physics, 21, 11 (1982).

7. A. Dip, Thesis (PH.D) North Carolina State University, 53, 11 (1993).

8. J. Nishizawa and T. Kurabayashi, J. Chrystal Growth, 99, p 525-529 (1990).

9. M. D. Vilisova, A. E. Kunitsyn, L. G. Lavarent'eva, V.V. Preobrazhenskii, M. A. Putyato, B. R. Semyagin, S. E. Toropov and V. V. Chaldyshev, Semiconductors, 36, 9 (2002).

10. M. Kawanaka and J. Sone, J. Chrystal Growth, 95, p 421-424 (1989). 